\title{
Case report of a pancreatic squamoid cyst
}

\author{
Dae-Gwang Yoo, Shin Hwang, Dae-Wook Hwang, Ki-Hun Kim, Chul-Soo Ahn, \\ Tae-Yong Ha, Gi-Won Song, Dong-Hwan Jung, Gil-Chun Park, and Sung-Gyu Lee
}

\author{
Department of Surgery, Asan Medical Center, University of Ulsan College of Medicine, Seoul, Korea
}

\begin{abstract}
Squamoid cyst of the pancreas is a very rare disease and it has been proposed only recently as a distinct pathologic lesion. We herein present a case of pancreatic squamoid cyst in a patient who underwent laparoscopic resection. A 60-year-old woman had an abdominal computed tomography (CT) scan for a routine check-up, and a multi-cystic lesion of $1.8-\mathrm{cm}$ in size was incidentally found in the tail of the pancreas. Biochemical laboratory tests were within normal limits. At first, we presumed that the most likely diagnosis of the cystic lesion was an intraductal papillary mucinous neoplasm. To treat this lesion, we performed laparoscopic spleen-saving distal pancreatectomy. The patient showed the usual routine postoperative course and she was discharged 10 days after surgery. On examination of the resected specimen, a well-defined, oligolocular cystic mass was found in the pancreatic tail, without a solid portion. Histologic examination revealed that the cysts had linings ranging from flat squamoid cells to transitional cells with non-keratinization. After immunohistochemical staining, the final diagnosis was confirmed to be squamoid cyst of the pancreas. This lesion appears to be regarded as a benign entity, thus an extended operation should be avoided and resection of the lesion can be performed minimally. (Korean J Hepatobiliary Pancreat Surg 2013;17:181-185)
\end{abstract}

Key Words: Pancreatic squamoid cyst; Pancreatic cancer

\section{INTRODUCTION}

Recently, the detection of cystic lesions in the pancreas has greatly increased because of the wide use of high-resolution diagnostic imaging techniques. ${ }^{1}$ In contrast to solid tumors which are usually ductal adenocarcinomas, which have a very poor prognosis, most cystic lesions of the pancreas are either benign or low-grade malignancies; thus, it is relatively easy to perform curative resection for them. ${ }^{2}$ Therefore, cystic and intraductal tumors of the pancreas consist of an essential category with a challenging differential diagnosis. ${ }^{3}$ Pancreatic squamoid cyst is defined as a lesion with cyst-like dilated ducts that are lined by non-keratinized squamous epithelium, and it is a recently recognized type of cystic lesion of the pancreas. ${ }^{2}$ We herein present a case of a patient with a pancreatic squamoid cyst who underwent laparoscopic resection.

\section{CASE}

A 60-year-old woman who underwent abdominal computed tomography (CT) for a routine health check-up was found to have a $1.8-\mathrm{cm}$ cystic lesion in the tail of the pancreas. The patient was admitted to our center for further examination. She had no symptoms related to the lesion. Her abdomen was soft and flat, and no tenderness or rebound tenderness was observed. Complete blood count, electrolytes, and liver function tests were within normal limits. There was no evidence of pancreatitis. Both the serum carcinoembryonic antigen (CEA) and carbohydrate antigen 19-9 (CA19-9) levels were normal. A dynamic CT of the pancreas showed multiple cystic lesions in the tail of the pancreas and the largest cystic lesion showed a lobulated contour without communication with the pancreatic duct. The lesion measured $2.2 \mathrm{~cm}$ in maximal diameter and had several small calcifications. There were no indications of invasion into the surrounding tissues or dilatation of the main pancreatic duct (Fig. 1A and

Received: October 15, 2013; Revised: October 21, 2013; Accepted: November 1, 2013

Corresponding author: Shin Hwang

Department of Surgery, Asan Medical Center, University of Ulsan College of Medicine, Poongnap-dong, Songpa-gu, Seoul 138-736, Korea Tel: +82-2-3010-3930; Fax: +82-2-3010-6701; E-mail: shwang@amc.seoul.kr 

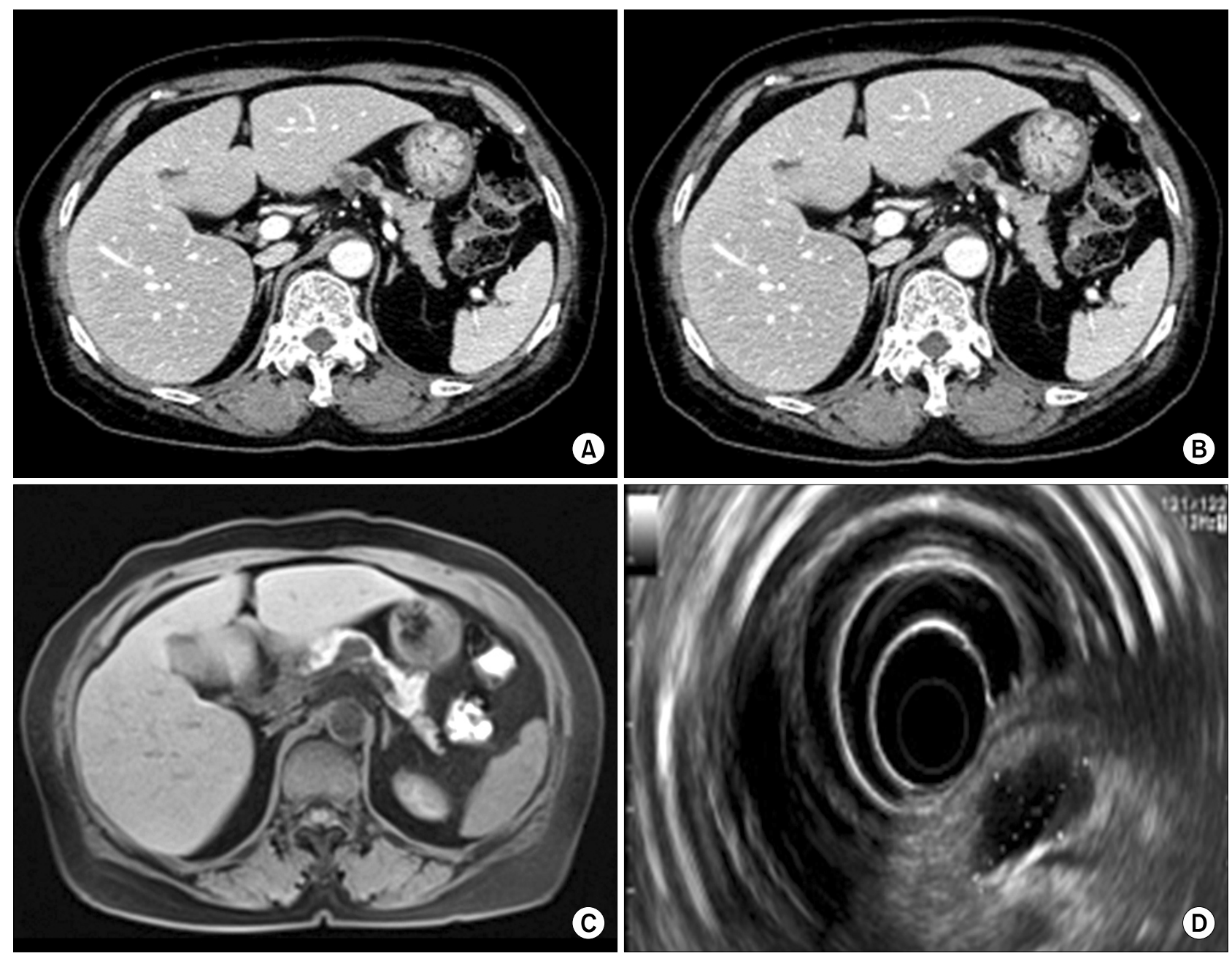

Fig. 1. Pancreas dynamic CT showed cystic lesions (A and B). The largest lesion measured $2.2 \mathrm{~cm}$. Magnetic resonance cholangiopancreatography demonstrated a $2.2-\mathrm{cm}$ round cystic lesion that did not communicate with the main pancreatic duct (C). Endoscopic ultrasonography showed a $2.2 \times 1.3 \mathrm{~cm}$-sized cystic lesion (D). The cystic lesion was divided into several cysts by thin septa.
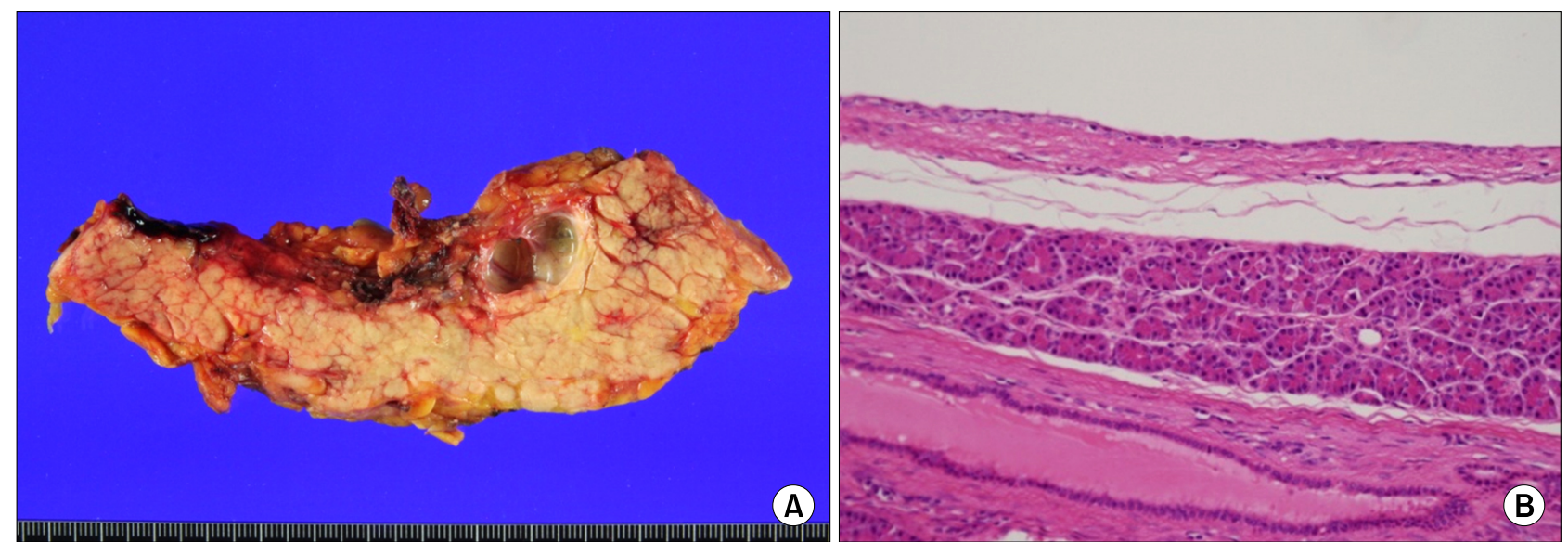

Fig. 2. Photograph of the resected specimen showing a multilocular cystic lesion with thin septa (A). Microscopically, the cyst wall was lined with nonkeratinized squamous epithelium (B). There were mucoproteinaceous dense eosinophilic materials in the cyst lumen $(\mathrm{HE} \times 100)$. No tall columnar mucinous cells were identified. 
1B). Magnetic resonance cholangiopancreatography revealed a $2.2-\mathrm{cm}$ round cystic lesion in the tail of the pancreas which had no communication with the main pancreatic duct. The main pancreatic duct was not dilated (Fig. 1C). Endoscopic ultrasonography revealed a $2.2 \times 1.3$ cm-sized cystic lesion and a $5 \times 2.5 \mathrm{~mm}$-sized mural nod- ule; the septation within the cyst and the cyst wall was thin; and the cyst was well-demarcated from the surrounding tissues. The pancreas showed normal parenchymal echogenicity and the main pancreatic duct had a normal caliber (Fig. 1D). We diagnosed the cystic lesion as an intraductal papillary mucinous neoplasm.
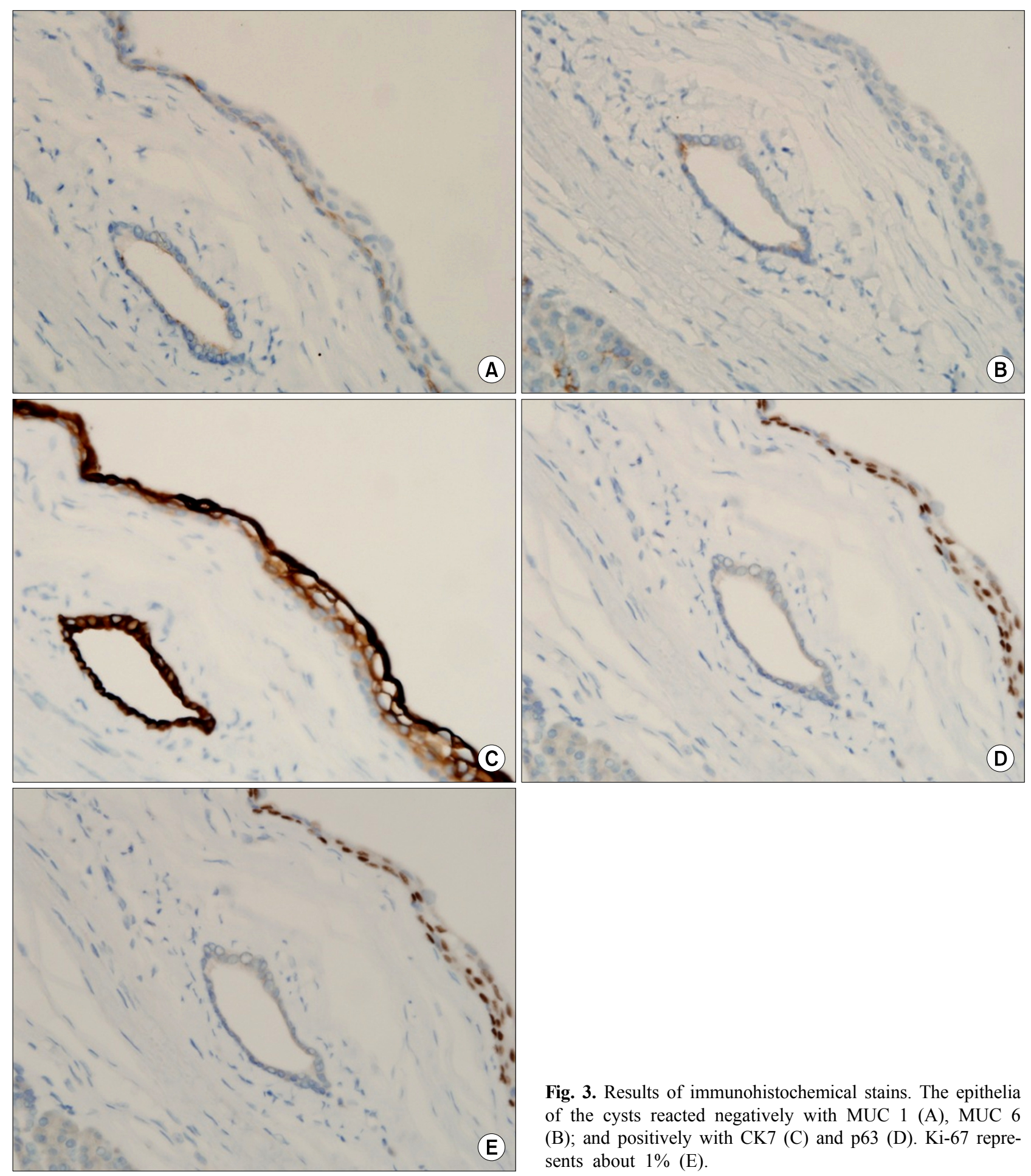

Fig. 3. Results of immunohistochemical stains. The epithelia of the cysts reacted negatively with MUC 1 (A), MUC 6 (B); and positively with CK7 (C) and p63 (D). Ki-67 represents about $1 \%(\mathrm{E})$. 
To treat this lesion, we performed laparoscopic spleensaving distal pancreatectomy. During the operation, the cystic lesion, approximately $3 \mathrm{~cm}$ in diameter, was found arising from the border between the body and tail of the pancreas without any infiltration into the surrounding tissues. Most of the lesion was located within the pancreatic tissue, and its border was well-defined. There was no peripancreatic lymph node enlargement. The preplanned surgery was performed uneventfully. The patient showed the usual routine postoperative course and was discharged 10 days after surgery. The patient quickly returned to her normal activities.

On examination of the resected specimen, the cystic lesion of the pancreas $(2 \times 1.8 \times 1.5 \mathrm{~cm})$ was multi-locular with thin septa (Fig. 2). A well-defined, oligo-locular cystic mass was in the pancreatic tail without a solid portion. Inner surface of the cyst was smooth and glistening and the cyst contained serous fluid. No connection with the main pancreatic duct was identified. Histologic examination revealed that the cysts had linings ranging from flat squamoid cells to transitional cells with non-keratinization (Fig. 3A). No tall columnar mucinous cells were identified. No associated lymphoid or splenic tissue was present. The cyst walls were composed of relatively thin fibrous issue. The epithelia of the cysts reacted negatively with MUC 1 and MUC 6 (a marker for intercalated duct centroacinar cells), and positively with CK7 and p63, and negatively with inhibin. Ki-67 represented about 1\% (Fig. 3A to 3E). The results of these immunohistochemical stains are summarized in Table 1. The final diagnosis was confirmed as squamoid cyst of the pancreas.

\section{DISCUSSION}

In 2007, Othman et al reported on 6 cases of cystic lesions with distinctive morphologic, immunophenotypic,

Table 1. Summary of immunohistochemical stains

\begin{tabular}{ll}
\hline \multicolumn{1}{c}{ Marker } & Results \\
\hline CK7 & Positive \\
CK20 & Negative \\
p63 & Positive \\
MUC-1 & Negative \\
MUC-6 & Negative \\
Inhibin & Negative \\
Ki-67 & About $1 \%$ \\
\hline
\end{tabular}

and clinical characteristics, and proposed the term squamoid cyst of the pancreatic ducts to refer to these types of lesions. ${ }^{2}$ They mentioned that this type of cystic lesion typically resulted from uni-locular cystic dilatation of the ducts and had variable linings ranging from attenuated, flat, non-stratified squamous to transitional and stratified squamous epithelium, without a cornified layer or parakeratosis. ${ }^{2}$ However, no tall-columnar mucinous cells or acinar cells are evident in a squamoid cyst. ${ }^{1}$

The squamoid cyst of the pancreas is characterized by a flat, transitional, or squamous epithelium, and a p63 expressing basal and parabasal cell component. Kurahara et al mentioned that the microscopic version of this lesion is a relatively common incidental finding in the pancreas, and these, along with the expression of MUC 1 and MUC 6 , suggest that the process originates from the entroacinar/intercalated ductal system. ${ }^{1}$ However, the surface cells were negative for MUC1 and MUC6 in our case. So we suggest that the immunophenotyping of the squamoid cyst should be studied further, especially from the point of view of the expression of MUC1 and MUC6. The expression of p63 in the cells forming the basal region is essential because p63 is a transitional/squamous cell marker and is not detected in the normal pancreas or in non-squamous neoplasms. The surface cells were negative for MUC1 and MUC6 (markers present in intercalated duct cells) in this case. Thus, we were able to determine that squamoid cysts can be diagnosed without MUC1 or MUC6 being expressed.

There are other types of squamous-lined cysts of the pancreas, such as lymphoepithelial cysts, epidermoid cysts within an intrapancreatic accessory spleen, and dermoid cysts. $^{4}$ Lymphoepithelial cysts are lined by well-differentiated stratified squamous epithelium surrounded by a band of dense lymphoid tissue. ${ }^{4}$ Epidermoid cysts within the intrapancreatic accessory spleen are lined by attenuated squamous cells, usually non-stratified, surrounded by normal-appearing splenic tissue. ${ }^{5}$ Dermoid cysts are morphologically similar to teratomas seen in other sites. The presence of adnexa-type elements (sebaceous glands, hair, etc) is more typical of dermoid cysts. ${ }^{6,7}$

These cystic lesions of the pancreas are thought to be all benign. Clinically, it is important to distinguish squamoid cysts of the pancreatic ducts from mucinous cyst-forming neoplasias, especially mucinous cystic neo- 
plasms and intraductal papillary mucinous neoplasms. ${ }^{2}$ In contrast to squamoid cyst of the pancreatic ducts, which appears to be a benign lesion, these mucinous lesions have the risk of malignant transformation and have the potential to progress to invasive adenocarcinoma. However, the differential diagnosis of pancreatic cystic lesions is not easy because the radiologic and clinical findings are not specific.

Kurahara et al mentioned that tumor markers (CEA and CA19-9) may be high in squamoid cysts of the pancreas. ${ }^{1}$ We investigated 5588 patients who underwent a pancreatic biopsy or pancreatic resection from 2009 to 2012 in our institution, and found 7 cases of squamoid cysts of the pancreas. There were no connections to the main pancreatic duct and no solid portion in any of the cases. The levels of CEA, CA19-9 and CA72-4 were 1.86 \pm 0.91 $\mathrm{ng} / \mathrm{ml}, 19.55 \pm 26.9 \mathrm{U} / \mathrm{ml}, 2.57 \pm 0.23$, respectively. There was one patient whose CA $19-9$ was $76.6 \mathrm{U} / \mathrm{ml}$, but this was not a specific finding because a pancreatic intraepithelial neoplasia was also found in her pancreas biopsy report. ${ }^{8}$ Thus, we suggest that tumor markers can be an important method to use in the differential diagnosis. CEA is a very accurate tumor marker for diagnosing mucinous pancreatic cystic neoplasms, although the accuracy of this marker and the cut-off level vary among laboratories. ${ }^{9,10}$

In conclusion, squamoid cyst of the pancreas is a very rare disease, and it has been proposed only recently as a distinct pathologic lesion. However, the diagnosis of squamoid cyst will increase due to improvements in imaging techniques such as $\mathrm{CT}$ scanning and magnetic resonance imaging. This lesion is regarded as a benign entity, thus an extended operation should be avoided and resection of the lesion can be performed minimally.

\section{REFERENCES}

1. Kurahara H, Shinchi H, Mataki Y, et al. A case of squamoid cyst of pancreatic ducts. Pancreas 2009;38:349-351.

2. Othman M, Basturk O, Groisman G, et al. Squamoid cyst of pancreatic ducts: A distinct type of cystic lesion in the pancreas. Am J Surg Pathol 2007;31:291-297.

3. Brugge WR. The use of EUS to diagnose cystic neoplasms of the pancreas. Gastrointest Endosc 2009;69(2 Suppl):S203-S209.

4. Adsay NV, Klimstra DS, Compton CC. Cystic lesions of the pancreas. Introduction. Semin Diagn Pathol 2000;17:1-6.

5. Kanazawa H, Kamiya J, Nagino M, et al. Epidermoid cyst in an intrapancreatic accessory spleen: a case report. J Hepatobiliary Pancreat Surg 2004;11:61-63.

6. Adsay NV, Hasteh F, Cheng JD, et al. Lymphoepithelial cysts of the pancreas: a report of 12 cases and a review of the literature. Mod Pathol 2002;15:492-501.

7. Fernandez-Cebrian JM, Carda P, Morales V, et al. Dermoid cyst of the pancreas: a rare cystic neoplasm. Hepatogastroenterology 1998;45:1874-1876.

8. Klein WM, Hruban RH, Klein-Szanto AJ, et al. Direct correlation between proliferative activity and dysplasia in pancreatic intraepithelial neoplasia (PanIN): additional evidence for a recently proposed model of progression. Mod Pathol 2002;15:441-447.

9. Brugge WR, Lewandrowski K, Lee-Lewandrowski E, et al. Diagnosis of pancreatic cystic neoplasms: a report of the cooperative pancreatic cyst study. Gastroenterology 2004;126:1330-1336.

10. Cizginer S, Turner BG, Bilge AR, et al. Cyst fluid carcinoembryonic antigen is an accurate diagnostic marker of pancreatic mucinous cysts. Pancreas 2011;40:1024-1028. 